\title{
Cytotoxicity of Acrylic Resin: A Review
}

\author{
Arunasree V Mallikarjuna
}

\section{Introduction:}

Acrylic resins are the most widely used materials in dentistry. It is one of the oldest materials in use for the fabrication of denture bases. Acrylic resins are produced by polymerisation of methylmethacrylate, which is a monomer and is available as a transparent liquid. But there have been various reports on the cytotoxic effects of methylmethacrylate due to the leaching of the residual monomer from the polymerized denture bases. The aim of this article is to review the literature pertaining to the cytotoxic effects of methylmethacrylate and the methods of decreasing the concentration of residual monomer in denture bases.

\section{Method and Methodology:}

A review of literature from 1973-2013 was done. The articles were chosen based on their specificity about (1) cytotoxic effects of methylmethacrylate(2) hypersensitivity to methymethacrylate(3) the biodegradation of acrylic resin in the oral cavity(4) comparison of the concentration of residual monomer in different methods of polymerisation(5)methods of decreasing the residual monomer.

\section{Discussion:}

Acrylic resins are formed by polymerisation of methylmethacrylate. But this polymerisation does not take place completely, thereby leaving some amount of residual monomer within the denture base. This residual monomer starts leaching out over a period of time causing various cytotoxic effects on the adjacent tissues.

\section{Cytotoxic Effects of Methylmethacrylate:}

An in vitro study done in Portugal sheds light on the cytotoxic effects of methylmethacrylate on human gingival fibroblast cell line and human fetal lung cell line. This study reveals that there were changes in the viability of the cells depending on the concentration of the methylmethacrylate. Also, it was found that the human fetal lung fibroblasts were affected by the vapours of the methymethacrylate ${ }^{[1]}$. Another study done on three different cell lines( HeLa, N1H3T3 and Hep2) also revealed cytotoxic effects on the cell lines particularly the epithelial cell line. It was also noted in the study that the cytotoxity decreased when the resins were left to elite in cell line for a longer duration ${ }^{[2]}$.

A study done by Lai et al examines the cytotoxic potential of resin liquids of 3 in situ reclining polymers whose major component included methylmethacrylate(MMA). This study estimated the amount of MMA, IBMA and 1,6-HDMA using HPCL. Later cultures in primary human gingival fibroblasts and periodontal ligament were done and estimated by MTT assay which determines the Mitochondrial dehydrogenase, an indicator of cellular viability. Interestingly, it was found that 1,6-HDMA caused severe cytotoxicity whereas MMA had least cytotoxic effect. Most of these effects were death by necrosis rather than by apoptosis. The authors also go on to explain that the effect on the in vivo conditions may be far more greater than the controlled in vitro conditions ${ }^{[3]}$.

\section{Hypersensitivity To Methymethacrylate:}

A study done by Goncalves et al documents the allergic tendencies towards methylmethacrylate resins in a 60yr old lady despite the residual content of monomer being within the international standards. Also a patch test on the patient revealed positive reactions ${ }^{[4]}$. Another study brings to light the allergic reactions caused by methylmethacrylate through a case report. The study also involves a patch test on 3 different patients of whom 2 are dentists. The patch test was done for liquid monomer, autopolymerising resin and light-cures resins. The patch test revealed a severe positive reaction for liquid monomer, moderate positive reaction for autopolymerising resin and a mild positive reaction for light-cured resin on 2 of the 3 patients. The study also gives alternate methods for preparing interim crowns to decrease the residual monomer thereby preventing any allergic reactions ${ }^{[5]}$.

\section{The Biodegradation of Acrylic Resin In The Oral Cavity:}

A study on the biodegradation of acrylic resins in the oral cavity indicates that the biodegradation of acrylic resins depends on various factors like saliva, chewing, thermal and chemical dietary changes. The 
biodegradation of the acrylic resin leads to leaching of residual monomer which can cause cytotoxicity ${ }^{[6]}$. Another study evaluates the leaching of methylmethacrylate and formaldehyde in oral and artificial conditions. Interestingly, it was noted the formaldehyde was cytotoxic in its leaching concentration than the MMA. Also, pre-leaching of the acrylic resin in hot water $\left(50^{\circ} \mathrm{C}\right)$ decreases the cytotoxic effects in the oral cavity ${ }^{[7]}$. Another study, by Minoru Kawaguchi et al, evaluates the amount of leachable monomer from light-activated reline material. It compares the amount of leachable monomer with the duration of light exposure. It was found that prolonged light exposure decreased the amount of leachable monomer from the reline materials. Also, it was noted that the amount of leachable monomer increases with increase in thickness of the reline material ${ }^{[8]}$.

\section{Comparison of the Concentration of Residual Monomer In Different Methods of Polymerisation:}

A study by Kedjarune et al is a comparison of the amount of residual monomer content and the MMA in saliva after incubation, between 3 heat cure and 3 autopolymerised acrylic denture bases. It was found that the amount of residual monomer in cured resin was dependent on (1) type of polymerization(2) amount of mixture ratio (3) processing method (4) time of curing. Also cytotoxicity of MMA released in saliva was examined in human oral fibroblasts in vitro, and it was found that some types of acrylic resins are cytotoxic ${ }^{[9]}$. Another study also states that there is a decrease in the residual monomer concentration in acrylic resins polymerised by microwave irradiation than the conventional boiling water polymerisation ${ }^{[10]}$.

\section{Methods of Decreasing The Residual Monomer:}

A study by Jorge et al evaluates the effects of 2 post-polymerisation treatments and different cycles of polymerisation on the cytotoxicity of 2 denture base resins-Lucitone 550 and QC 20. While Lucitone 550 was processed by long/short cycles, QC 20 was processed by reverse/ normal cycle. Later they were divided into 3 groups: (1) microwaved (2) water-bath at $55^{\circ} \mathrm{C}$ and (3) no post-polymerisation. It was revealed that long cycle increased the cytotoxicity of Lucitone 550; post-polymerization with water-bath reduced the cytotoxicity of the long cycle processed Lucitone $550^{[11]}$.

Another study documents that the amount of residual monomer decreased by 4-fold when autopolymerised resin was subjected to microwave irradiation post-polymerisation ${ }^{[12]}$.

A study by Harrison A et al subjected 23 heat-polymerised denture base resins to different cycles of polymerisation. It was found that incubation in water at $70^{\circ} \mathrm{C}$ for $7 \mathrm{hrs}$ followed by immersion in water at $100^{\circ} \mathrm{C}$ for $1 \mathrm{hr}$ was the best method and there was minimal release of residual monomer ${ }^{[13]}$. In another study polymerization of autopolymerised resins at higher temperatures resulted in lesser concentration of residual monomer. Also the authors note that the amount of residual monomer varies with the ratio of polymer to monomer $^{[14]}$.

Interestingly, a study by $\mathrm{M}$ Vojdani et al shows that the immersion of heat-cured denture base resins overnight in resin cleansers like $1 \%$ sodium hypochlorte and alkaline peroxide solution increased the cytotoxicity of the resin. Also, immersion of the denture base in water decreased the cytotoxicity. These effects were significant only after $72 \mathrm{hrs}{ }^{[15]}$. Another study also states that the amount of residual monomer aries based on the ratio of polymer to monomer, the curing cycles and the methods of polymerization. The ratio 5:3 polymer to monomer is less cytotoxic than the ratio 4:3. It was also found that immersion of denture base in water for $24 \mathrm{hrs}$ post-polymerisation decreases the cytotoxicity ${ }^{[16]}$.

According to a study by Vanessa M Urban et al, water-bath post-polymerisation increases the degree of conversion of the monomer and decreases the leaching of residual monomer. One of the tested materials showed an increase in the flexural strength after water-bath ${ }^{[17]}$. Another study observed that the residual monomer content in denture reline materials decreased considerably after immersion in hot water post-polymerisation. The residual monomer in materials immersed in a water-bath was lesser than the materials irradiated with microwave. But compared to a non-treated denture reline material the amount of residual monomer was less in both the post-polymerisation methods ${ }^{[18]}$. In an another study it was found that the amount of residual monomer decreased with additional cycles of curing with microwave. A significant decrease was observed in light-cured resins after microwave irradiation for 5-10 minutes $^{[19]}$.

\section{Conclusion:}

(1) Acrylic resins are cytotoxic and have the tendency to cause hypersensitvity.

(2) The cytotoxic effect of Methylmethacrylate depends on the polymer:monomer ratio, duration of curing, method of curing.

(3) Post-polymerisation immersion of heat-cured resin in water at $50^{\circ} \mathrm{C}$ for $24 \mathrm{hrs}$ prior to insertion decreases the cytotoxicity.

(4) Post-polymerisation irradiation of autopolymerised resins in microwave decreases the cytotoxicity. 


\section{References:}

[1]. Cytotoxicity of acrylic based resin compounds in a human gingival fibroblat cell line. Rev Port Estomatol Med Dent Cir Maxilofac 2013;54(2):87-90

[2]. Cytotoxicity of two autopolymerised acrylic resins used in orthodontics. Tatiana Siqueira Goncalves et al; Angle orthodontist2008; 78;5:926-30.

[3]. Cytotoxic effects of dental resin liquids on primary gingival fibroblasts and periodontal ligament cells in vitro. Lai et al; Journal of oral Rehabilitation; 2004; 31;1165-72.

[4]. Allergy to auto-polymerized acrylic resin in an orthodontic patient; Goncalves et al; AM J Orthod Dentofacial Orthop 2006; 129431-5.

[5]. Hypersensitivity to methyl methacrylate: Mode of treatment Nira Hochman and Maya Zalkind; J Prosthet Dent 1997; 77:93-6.

[6]. Biodegradation of acrylic based resins: A review Ana F.Betten court et al: Dental materials 26 (2010) e 171-e180.

[7]. Leaching and cytotoxicity of formaldehyde \& Methyl methacrylate from acrylic resin denture base materials: Hironori T suchiya et al: J Prosthet Dent 1994; 71: 618-24.

[8]. Effect of light-exposure duration on the amount of leachable monomers from light-activated relive material: Minoru Kawaguchi et al: J Prosthet Dent 1996; 75: 183-7.

[9]. Release of Methyl methacrylate from heat-cured and autopolymerised resins: Cytotoxicity testing related to residual monomer; Kedjarune et al; Australian Dental Journal 25-30.

[10]. Comparison of denture acrylic resins cured by boiling water \& microwave energy, Trnong et al; Aus Dent J; 1988; 33:201-4.

[11]. Biocompatibility of denture based acrylic resins evaluated in culture of L929 cells. Effect of polymerization cycle and postpolymerization treatments; Jorge et al; Gerodontology; 2007; 24; 52-57.

[12]. Microwave polymerization of denture base materials. A comparative study ; Blagojevic V, et al; J. Oral Rehabil 1999; 26: 804-8.

[13]. 13.Effect of the curing cycle on residual monomer levels of acrylic resin denture base polymers: Harrision. A et al; J. Dent 1992; 20: $370-4$.

[14]. The effects of process variables on levels of residual monomer in autopolymerising dental acrylic resins: Lamb DJ et al; J. Dent 1983; 11: 80-8.

[15]. Cytotoxicity of Resin - Based cleansers: An in Vitro study: M Vojdani et al; IRCMJ 2010; 12(2): 158-162; Iranian Red Crescent Medical Journal.

[16]. The residual monomer in dental acrylic resin and its adverse effects; Nedeljka Ivkovic et al; Contemporary materials IV -1 : 2013: 87-91.

[17]. Effect of water-bath post-polymerization on the mechanical properties, degree of conversion, and leaching of residual compounds of hard chairside reline resins: Vanessa M. Urban et al; Dental materials; 25; 2009: 662-671.

[18]. Residual monomer of reline acrylic resins. Effect of water-bath and microwave post-poly treatments; Vanessa M. Urban et al: Dental materials 23: 2007; 363-368.

[19]. The Effect of Additional Microwave curing cycle on Residual Monomer Release from Different Acrylic Resin Materials; Omar A Sheet et al; Al-Rafidain Dent J; 2011; 11: 397-403. 